\title{
ON THE USE OF COLOUR IN EXPERIMENTAL FLUID MECHANICS
}

\author{
J.T. TURNER \& S. ZHANG \\ School of Mechanical, Aerospace and Civil Engineering, University of Manchester, Manchester, UK.
}

\begin{abstract}
This paper discusses how colour is used in experimental fluid flow studies. Firstly, colour (defined scientifically by the wavelength of the light) can be used to discriminate between different channels in optical instrumentation, making it possible to measure several flow properties, simultaneously, and without any intrusion into the flow. These aspects of modern flow measurement are discussed in terms of some examples. Alternatively, the results of the flow measurement may be represented in the form of images, graphs and three-dimensional schematics with colour being used to identify and emphasise particular features that might otherwise remain concealed. The experimental results presented in the paper confirm that using colour to discriminate between sets of data has several important advantages over the older black and white or grey scale forms of representation. Not only do colours provide an excellent basis for communication through graphical and three-dimensional presentations, but the resulting clarity may also help to reveal complex forms of fluid flow behaviour. Moreover, the inherent artistic appeal of some colour representations can draw the viewer into the technical detail, helping to simplify physical phenomena in ways that would not otherwise be possible. Although the observations made on the presentation of data are illustrated with reference to a number of experimental fluid flow problems, many of the methods and observations would be applicable in other situations, where scientific or technological data needs to be presented.
\end{abstract}

Keywords: colour, data presentation, experiment, fluid flow measurement, graphics, images, light, understanding, wavelength.

\section{INTRODUCTION}

Turner and Zhang [1] discussed the arrival of the digital computer in a previous paper, highlighting the impact of digital methods since the first computers arrived in the mid-1950s. The paper also traced the development of digital technology, covering the introduction of hardware systems and the associated software. Several experimental flow problems were introduced and the data derived from these were used to show the benefits offered by coloured presentations, and other advanced forms of data processing.

In reviewing the rapid growth to dominance of digital technology, it needs to be understood that the innovations represented the result of research within many different organisations and were introduced in an entirely haphazard fashion. Thus, although the new computers permitted massive amounts of calculation to be performed routinely by the mid-1950s, these operations were much less convenient than is the case with modern systems. Moreover, the associated display devices (i.e. printers and monitors) and general purpose software packages for data processing took much longer to reach the mass market. As a result, coloured graphics and the colour monitor did not become common until 1984 with the arrival of the Apple Macintosh. Similarly, printers were limited to black and white operation until the 1990s. Naturally, for several years after their introduction, the cost of each of these tools was considerably higher than is now the case. Compare this with the situation today, where dedicated software tools (e.g. for word processing, statistical analysis, three-dimensional drawing and graphical presentation) are readily available to everyone, and at relatively low prices.

The ability to capture information on physical systems (and particularly fluid flow data) at very high speed could never have been envisaged by previous generations. Instead, the flow would be observed and reported in simple, easily understood, terms, such as a sketch, a measured flow rate or an averaged velocity. Figure 1, for example, shows some typical results presented by Leonardo da 

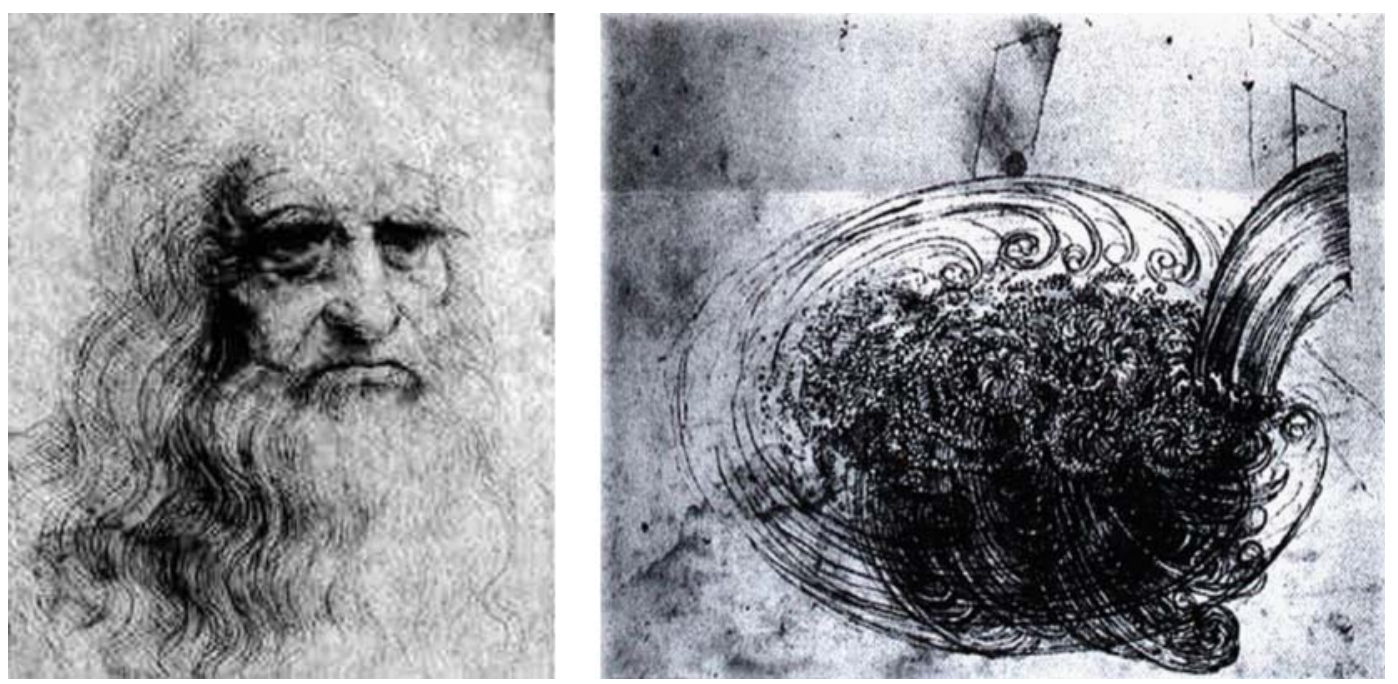

Figure 1: Leonardo da Vinci presented his results, showing the surface disturbances produced by water flowing into a distribution chamber, as a series of black and white sketches.

Vinci (1452-1519) [2]. Da Vinci studied the flow of water into a distribution chamber and reduced his results to a series of black and white sketches, indicating how the surface of the water in the chamber was disturbed. These observations on the behaviour of a turbulent jet, and the manner in which turbulent eddies are formed and move in the body of the flow, are still recognised as valid today, despite the lack of instrumentation in those early days. Imagine how coloured presentations would have added to the impact of Da Vinci's already considerable influence on experimental fluid mechanics.

Noting the current importance of digital technology, it is perhaps surprising to recall that the electronic calculator and the slide rule were still the most commonly used methods for large-scale calculations until the late 1970s. Contrast this with the situation today, where everyone can have desk top access (at relatively low cost) to a personal computer (PC). Moreover, the associated peripherals, such as the colour printer, mouse, keyboard and massive storage capacity, are ubiquitous, relatively low cost and demanded as a right by virtually every PC user. These developments have provided enormous benefits for the experimentalist, because of the many improved forms of instrumentation and data capture systems, together with more sophisticated methods of analysis and presentation, that are available.

In the present context, it is worth reflecting on the fact that the first digital computer only became operational in 1948. Since then, there have been continuing developments to the extent that digital technology now embraces not just computers but everyday devices, such as digital calculators, watches and clocks, controllers for central heating systems and other domestic appliances, such as microwaves and washing machines, digital cameras and scanners, mobile phones, digital video discs, personal music players, the internet and other devices too numerous to mention here. It is astounding that these devices only became available within the last twenty years [1].

Consider, now, the use and advantages of colour in measuring fluid flow properties, then the need to record and present these. Tremendous improvements in instrumentation have occurred as a result of the digital revolution, enabling important properties, such as the pressure, temperature and velocity of the fluid to be measured, often remotely using non-intrusive diagnostic methods, in ways that were not 
available previously. Earlier investigators such as Da Vinci had to rely on observation or, much later, could resort to physically intrusive methods in which a sensor (e.g. a thermocouple) was inserted into the flow, introducing the possibility that the flow patterns would be altered by the sensor. Now, many of these intrusive methods have been superseded by non-intrusive sensors and digital instrumentation systems, inevitably with a digital computer controlling the data acquisition and processing - please refer to [1] for more detailed discussion and a chronology of these developments.

Many of the measurement techniques introduced in the past twenty years make use of the principals of optics and rely, more often than not, on the properties of the light emitted by a laser (light amplification by stimulated emission and radiation). After over fifty years of intensive development, the name laser is now applied to a whole family of light-emitting devices, following the introduction of the first laser in the mid-1950s [3, 4]. The principal characteristic of a laser is its ability to produce an intense beam of light at a precisely specified wavelength that is collimated so that the diameter of its crosssection remains the same over very long distances. It also has other important properties that are exploited in measurement systems, although these properties cannot be discussed in any detail here.

Soon after the introduction of the laser, a range of non-intrusive flow velocity measuring techniques began to emerge, of which the first was laser Doppler anemometry (LDA). This was described by Yeh and Cummings [5] but more complete details have been given by Durst et al. [6] and Albrecht et al. [7]. The first LDA systems used a helium-neon laser operating at $633 \mathrm{~nm}$ (red) and were capable of measuring one (or sometimes two) components of the fluid velocity, simultaneously, and at a well-defined position in the flow.

With the arrival of the argon-ion laser (1964), coupled with improved optical components, such as colour filters and detectors, it became possible to generate a beam of light containing two or more colours simultaneously. Specialised instruments (e.g. the photometer and the spectrometer) can then be used to discriminate between these 'colours' to a fraction of a nano-metre. These developments allowed several channels of optical instrumentation to operate in parallel, with separation of the channels being made possible by colour filters. After some forty years, colour separation remains at the heart of all modern LDA systems, as will be demonstrated later in this paper.

Following intensive development, LDA and several other related optical diagnostic methods employing a laser as the light source are currently used to measure, for example, the fluid velocity, temperature and, simultaneously, the size and velocity of fluid borne particles.

In addition to point measuring techniques, such as LDA, both qualitative and quantitative methods based on digital imaging are increasingly used. These methods use high speed imaging to measure the movement of small particles carried by the flow. The most commonly used method is currently referred to as particle image velocimetry, or PIV, [8,9]. Digital imaging techniques not only offer considerable power and flexibility but also provide an opportunity for post-processing the images so as to enhance any chosen feature. No-where is the traditional view, that 'every picture is worth a thousand words', more appropriate than in experimental fluid flow studies. Thus, many measurements are made by capturing the movement of small particles (typically $5 \mu \mathrm{m}$ in diameter, or less) carried by the flow. Then, by using one or more specially designed digital cameras, and image analysis techniques, the distribution of the fluid velocity across a plane in the flow defined by a sheet of laser light can be obtained. Similarly, the distribution of the local fluid temperature can be measured by interrogating the images of small temperature sensitive liquid crystal particles that are carried by the flow. The wavelength (or colour) of the light reflected by these crystals changes in response to the fluid temperature. More information on this technique is available in [10]. Another family of spectroscopic methods relies on the detection and analysis of the wavelength distributions of the light emitted by the target substance, after irradiation by a laser source. These light scattering methods can be employed to measure the chemical composition of a fluid, or the concentration of combustion products in flue gases. 


\section{COLOUR AND ITS REPRESENTATION}

Vision is the term used to describe the ability of the eye to respond to electromagnetic radiation. By definition, light is that part of the electromagnetic spectrum that stimulates the retina of the (human) eye, and the brain, then associates different colours with different wavelengths of the light. Typically, the human eye has the ability to resolve wavelengths extending from the ultra-violet (around $380 \mathrm{~nm}$ ) to the infrared region of the visible spectrum (around $740 \mathrm{~nm}$ ). Even so, colour is subjective because the sensitivity to different wavelengths varies significantly between individuals, implying that each person sees a slightly different image when receiving the light scattered or reflected from an object. For the average subject, however, the sensitivity of the normal human eye varies in an approximately Gaussian fashion in daylight, reaching a maximum in the green region of the spectrum. Typically, thousands of different colours can be distinguished. However, modern optical devices show even greater sensitivity and better discrimination between wavelengths than this. More information can be found in [9b and c].

As discussed previously in [1], entirely new technical challenges needed to be overcome before the introduction of colour monitors and printers. In simple terms, a coloured image, for example, captured on a digital camera, is stored as an array of small coloured dots (or pixels). These coloured dots are represented, first as an electrical charge on the imaging device in response to the characteristics of the incident light, before being transmitted to the digital computer, where both the colour and position of the pixels within the image are stored numerically. When it becomes necessary to display the image, the stored data is converted back into a visual display through a colour printer, the computer monitor or the camera display screen. This ability to represent images in digital form has proved to be extremely valuable in experimental fluid mechanics and, as mentioned previously, several techniques are now based on digital imaging $[8,10,11]$. Furthermore, the rise in storage capacity, now typically measured in Giga-bytes $\left(10^{9}\right.$ bytes, where one byte represents one 16 bit number in binary form), has been accompanied by a massive growth in the image sizes offered by modern cameras (typically $10 \mathrm{MB}$ ). Similarly, the number and range of possible colours that can be represented digitally has risen steadily as the numerical precision of digital computers has increased, and corresponding improvements have been made to display monitors and colour printers. Again, these factors were discussed in the earlier paper.

In modern measurement systems, a PC is typically used to capture and store the data in digital form. The sensor signals can then be converted from the original input (say a temperature or pressure) into a voltage. These voltage values are then interpreted through detailed analysis, or the observed signals might need to be converted into another form, for example, back into the original pressures or temperatures. Specialised software also allows the data to be analysed, for example, to determine how the intensity of the scattered light varies with the wavelength. These advances have revolutionised experimental study, greatly extending the range of properties that can be measured, and enabling better methods of analysis and presentation to be adopted.

\section{SIMPLE GRAPHICAL DISTRIBUTIONS - THE ADVANTAGES OF COLOUR}

The most frequently used form of data presentation will consist of a graph showing how one variable changes as the other is altered. Superimposing several distributions on the same graph enables the effect of two (or more) 'independent variables' on the 'dependent variable' to be shown. Formerly, until colour techniques and hardware became generally available, graphs of this kind had to be produced in black and white. This created problems which were usually solved by allocating one symbol to each curve, for example, $+\times 0 \Delta$, possibly combined with dashed, chain-dotted, and continuous lines of varying thicknesses. This was a cumbersome process, in comparison with typical graphs today, where colour is conveniently used to distinguish between the curves. 
The results presented in Figs 3 and 4 show the acoustic signal produced by a microphone inserted into the wall of a pipe, so as to be exposed to the gas within, in response to an injected pulse of sound, for example, from a loudspeaker. In these experiments, the pipe contained air at atmospheric pressure but could have contained any other gas. Using this technique, known as acoustic reflectometry, an acoustic pulse is transmitted through the fluid in the pipe, and any reflections are recorded by a microphone or pressure transducer exposed to the same fluid. In this situation, the microphone responds to all the acoustic reflections produced by the internal features of the pipe, for example, small steps in the wall due to flanges, junctions, bends and valves. It is found that the reflections also carry information about any leakage through the pipe wall, or a blockage, irrespective of whether that blockage is caused by a liquid or a solid deposit. This provides an effective method for monitoring the condition of pipelines, such as those delivering natural gas from undersea wells [12]. During the development of this technique, experiments were performed to measure the acoustic response associated with a pool of water forming in a pipe filled with air (Fig. 2).

The time-wise variation of the small electrical output signals generated by the microphone were captured [via an analogue to digital converter (ADC)] and input to the PC. These signals were then processed to produce graphs of the type shown in Fig. 3. The figures on the right side of the graph represent the extent of the 'water blockage', as a percentage of the cross-section of the pipe, and the letters denote different positions along the length of the pool of water as the depth was altered. In producing the graph of signal amplitude against distance, the time required for the reflection caused by the water blockage to reach the microphone was converted into a distance by multiplying by the known speed of sound. From the acoustic response, the position and length of the blockage could then be determined $[12,13]$.

To obtain these results, the ADC was used to sample the microphone signal at a sampling rate of $25 \mathrm{kHz}$ (i.e. 25,000 voltage values were recorded and stored, in digital form, each second).

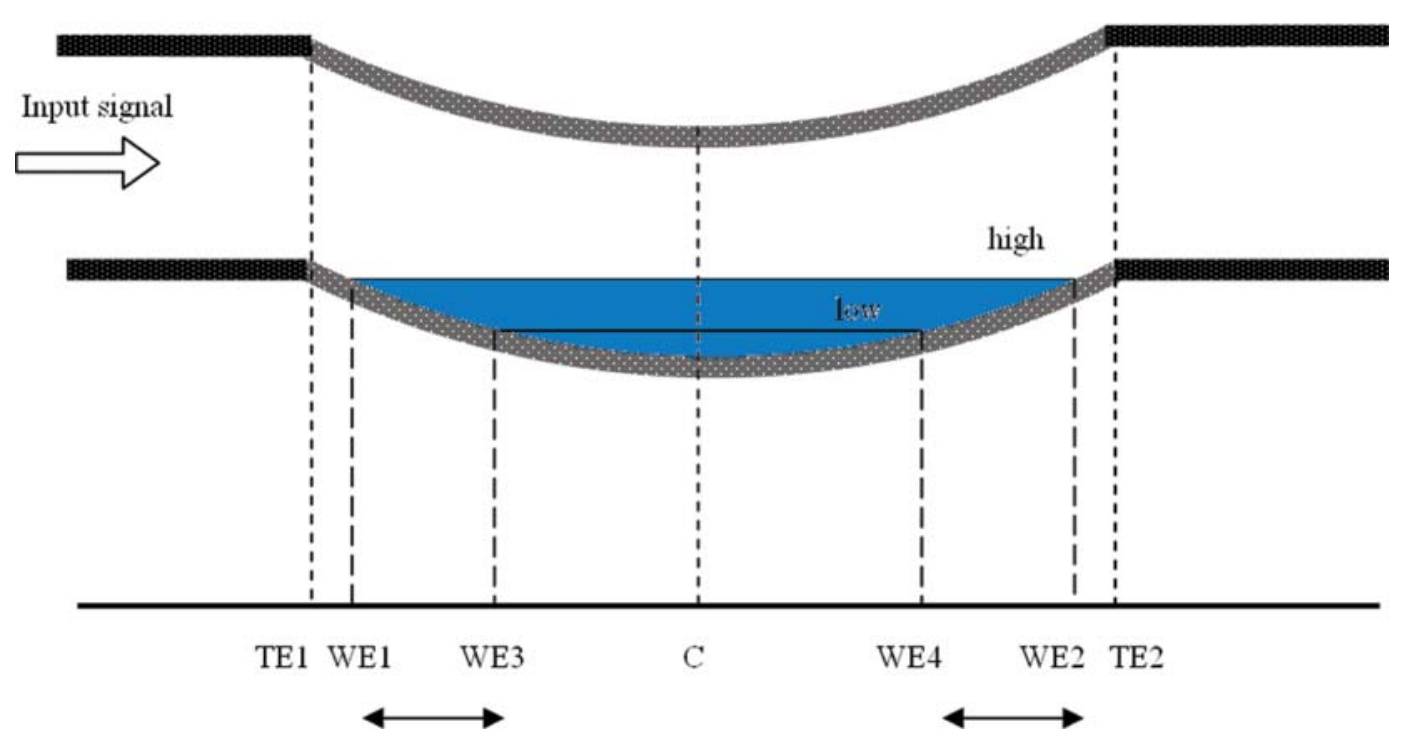

Figure 2: Schematic diagram showing the test arrangement used to investigate the acoustic signature produced by a pool of water in a pipe. 


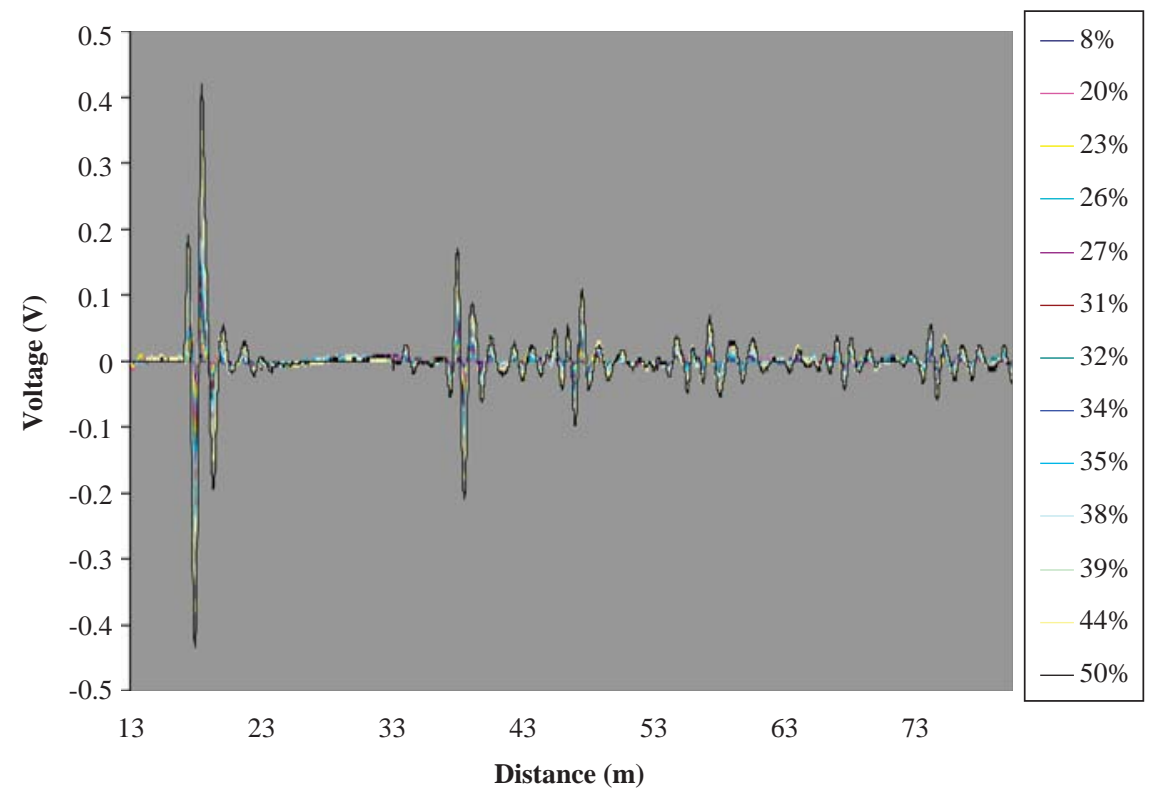

Figure 3: Time history of the acoustic signal, showing the position of a water pool inside a pipe.

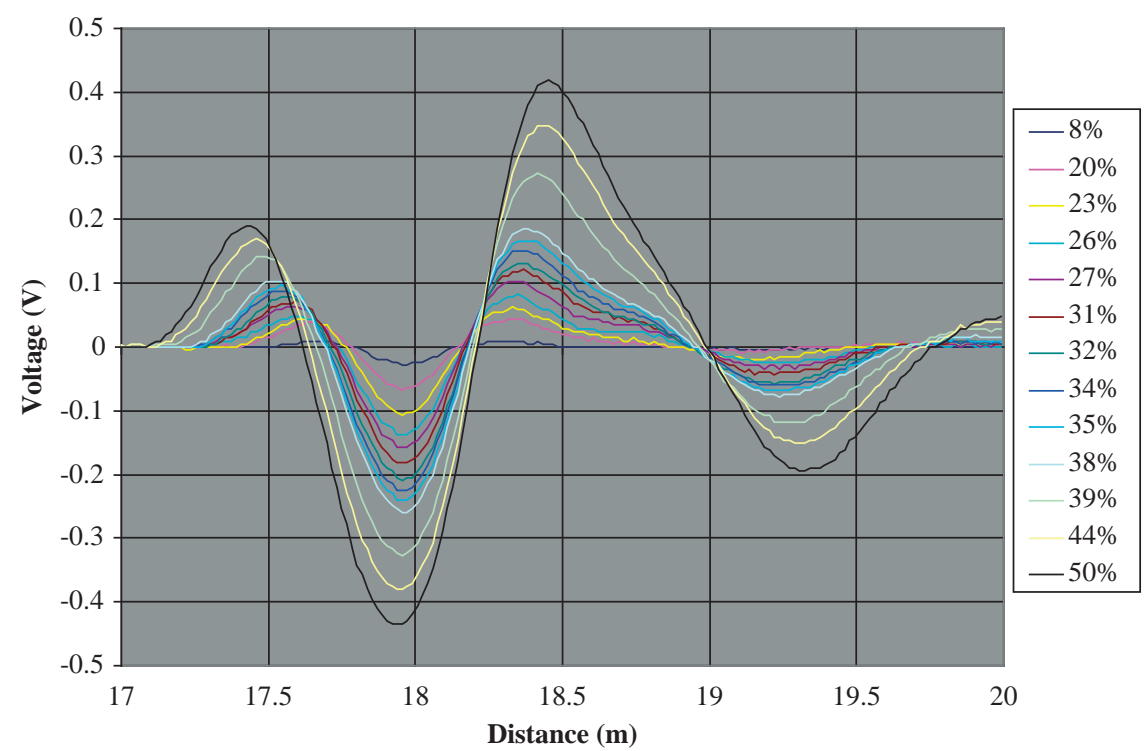

Figure 4: Typical processed data showing the first significant acoustic peak associated with the reflection caused by the water, for various amounts of blockage.

This relatively high sampling rate enables the scale representing the distance travelled by the acoustic reflections to be expanded without any loss of definition, thereby zooming in on the acoustic reflections caused by the water blockage. This expanded view of the data, in the vicinity of the acoustic reflection caused by the water blockage, is shown in Fig. 4. 
Apart from the visual appeal of this graphical display, observe how easy it is to follow any of the curves through from the left to the right of this graphical presentation. Clearly, assigning a different colour to each blockage condition enables the results to be interpreted very easily. This would not be the case for a black and white graph of this complexity.

The second example of multi-variable graphical representations, which will be referred to here, is taken from an experimental study of a turbulent jet of fan-blown air issuing horizontally from a precisely manufactured, axisymmetric nozzle. Complete details of this experimental programme may be found in [14], and a brief description is given in [1].

Firstly, the flow was 'visualised' by injecting paraffin vapour 'smoke' into the annular flow to reveal the flow patterns in the shear layers at the periphery of the main jet. These flow patterns were illuminated by means of a light sheet produced by a continuous wave argon-ion laser operating at $514.5 \mathrm{~nm}$ (i.e. in the green region of the spectrum). The light sheet was positioned to intersect the jet cross-section normal to its axis; in other experiments, not considered here but described in [1], another light sheet was positioned along the vertical diameter parallel to the axis of the jet.

In a second series of experiments, two-component LDA was used to obtain detailed quantitative measurements of the axial and radial velocity variations at successive stations downstream as the jet spread into the laboratory. The main features of the experimental arrangement are shown in Fig. 5, and have been described previously in $[14,15,16]$.

In these experiments, the LDA traversing system enabled the beams produced by an argon-ion laser to be brought to an intersection, and then positioned at each measurement position across the diameter of the jet. The measurement volume - defined by the crossing of the four intersecting laser beams shown in Fig. 5 - was traversed across the jet and local values for the axial and radial velocity components were obtained by operating the LDA instrumentation system. This traversing and measurement could be done automatically, under computer control, with a positional accuracy of better than $0.05 \mathrm{~mm}$ and a typical interval between the measurement positions of $2.5 \mathrm{~mm}$. During data collection, the laser light scattered

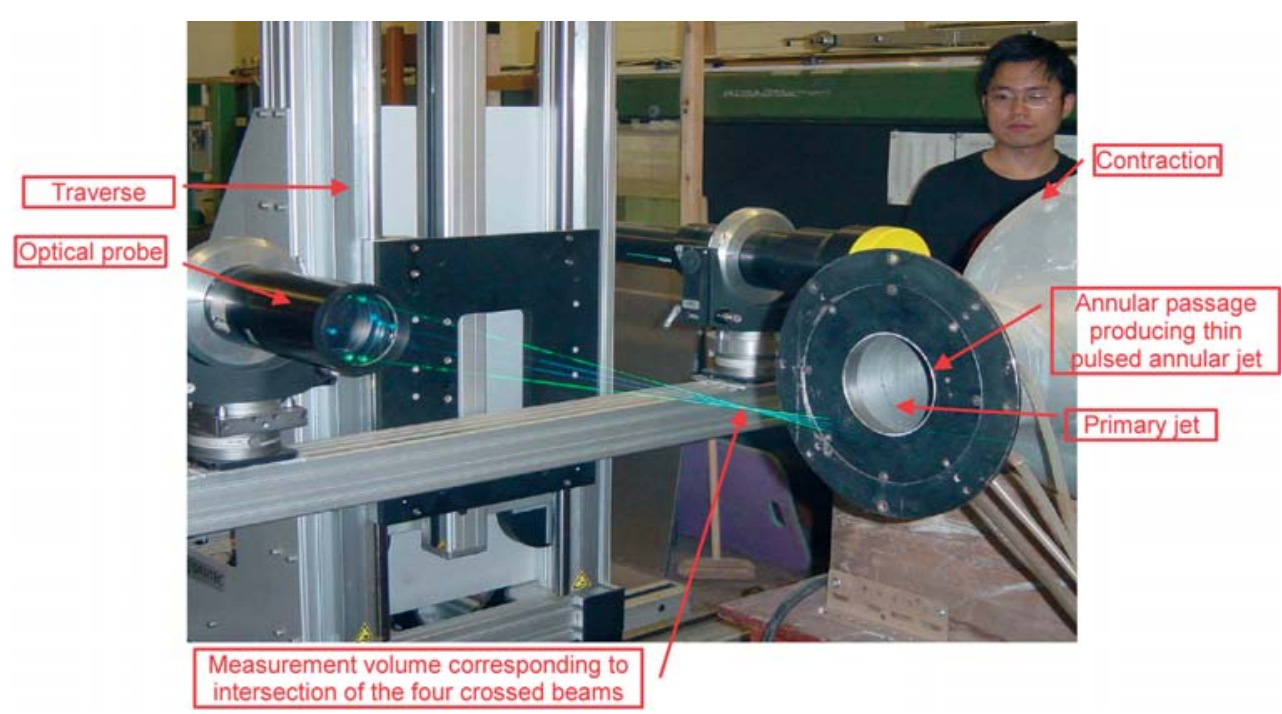

Figure 5: The free jet apparatus showing the second author in the process of data collection, the primary and secondary nozzles, parts of the two-component LDA apparatus and the highprecision automated traversing mechanism. 


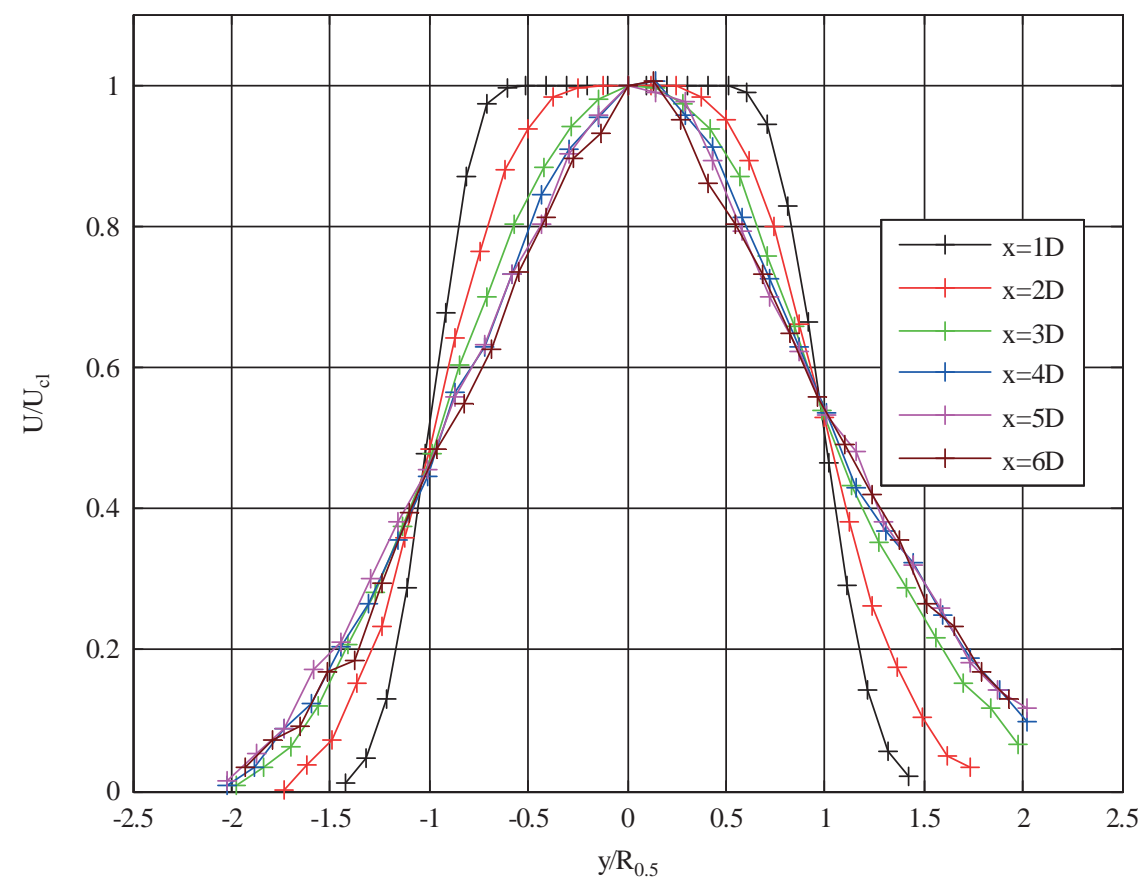

Figure 6: Typical distributions of the axial velocity at successive axial positions to show the development of the excited free jet for one flow rate and one excitation condition.

by small particles carried by the flow was separated into the original blue and green components, then captured on photo-detectors and converted into velocity information for the axial and radial directions by dedicated digital processing hardware. Figure 6 presents a small sample of the processed data, indicating how the distribution of the axial velocity component, measured in the vertical plane, changed as the free jet developed and merged with the laboratory atmosphere.

Figure 6 reveals some of the advantages of colour graphics, in particular, indicating how colour can be used to distinguish more clearly between the different distributions. Before the arrival of computerbased colour graphics, complex graphical information of this type was inevitably presented in a black and white (or, later, a grey scale) format. In this form, the data always lacked the visual impact of a colour display, and certainly never managed to convey the principal features with the same clarity.

\section{SOME EXAMPLES OF IMAGING}

To illustrate how digital imaging techniques can be utilised, reference will again be made to the jet flow experiments described earlier. In this case, the argon-ion laser was now used to produce a light sheet. This was transmitted across the jet flow, normal to its axis and the small annular jet at the periphery of the primary jet was identified by means of the paraffin 'smoke'. Images of the flow patterns revealed by this technique were recorded on a digital video camera at 25 frames/s (or $25 \mathrm{fps}$ ). The view in Fig. 7 represents a typical image captured on one video frame.

The flow visualisation reveals the behaviour of the shear layers which control the development of the excited turbulent jet. Specifically, Fig. 7 shows the shape of the jet cross-section at one instant while Figs 9 and 10 indicate how the shape of the shear layer changes with time, using false colour to provide better visual separation of the individual video images recorded at $25 \mathrm{fps}$. Here, alternate 


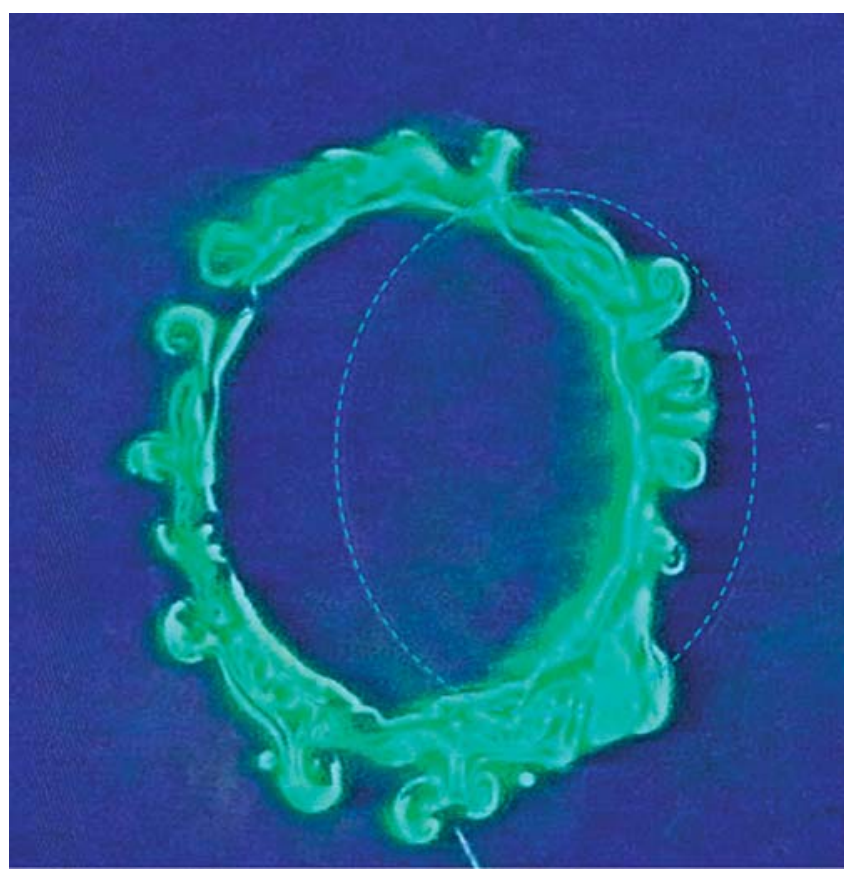

Figure 7: An instantaneous view of the cross-section of the excited jet. The light sheet, produced by an argon-ion laser operating in the green region of the spectrum, was aligned normal to the axis. The dashed line in the background is used to emphasise the position of the outlet plane of the primary nozzle.
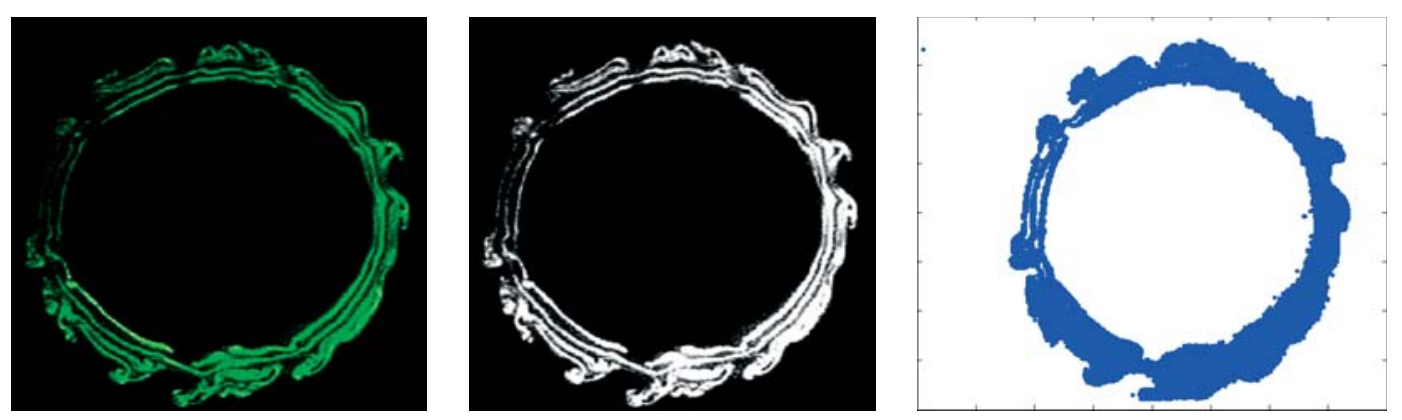

Figure 8: (a) Original view - as illustrated by Fig. 7. (b) Changed to a grey-scale image. (c) Each video 'slice' is assigned a (false) colour to distinguish it from its neighbours.

frames of the digital video record have been selected, to give a longer time interval (of $0.08 \mathrm{~s}$ ) between successive frames. The sequence of images was then processed in the manner shown in Figs 8-10 to reveal the behaviour of the pulsating shear layers produced by the aerodynamic excitation, obtained by pulsing the annular jet at the periphery of the primary jet.

Finally, it is worth noting that the montages in Fig. 10 were produced using the Matlab software package, offering the considerable advantage that the views can be rotated, with relative ease. 


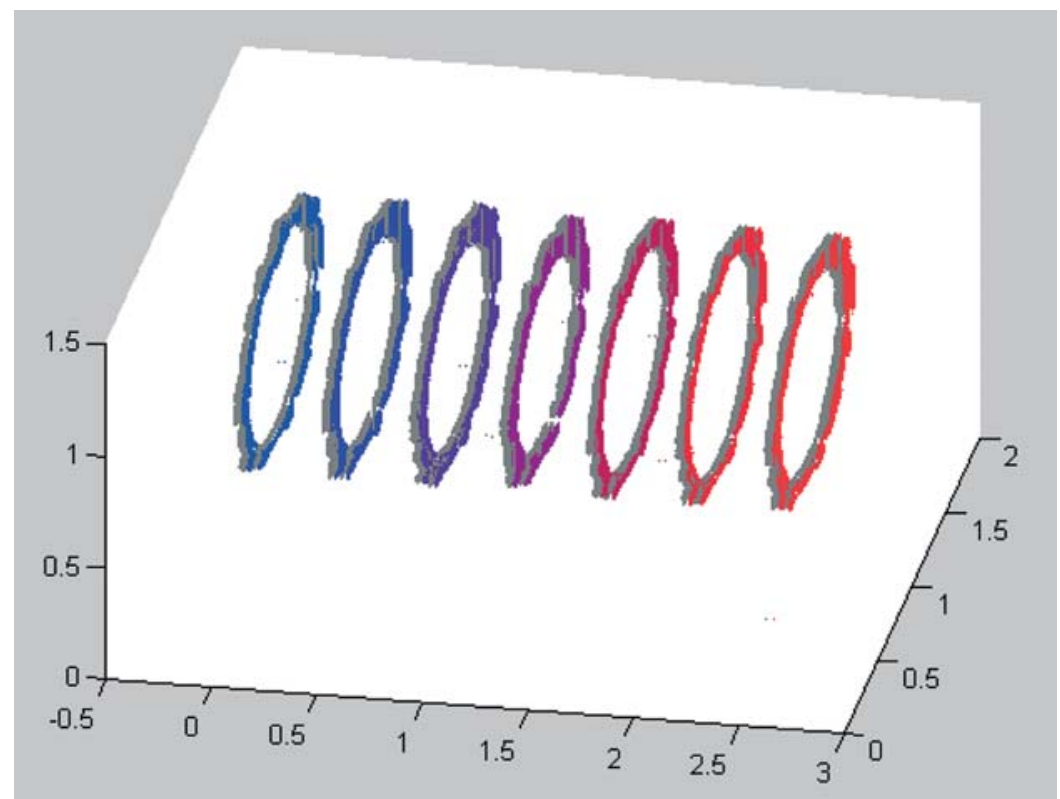

Figure 9: Successive slices are aligned along the chosen direction, separated by the assigned colour and an arbitrary distance (which might be dependent on the flow velocity or the frame time interval).

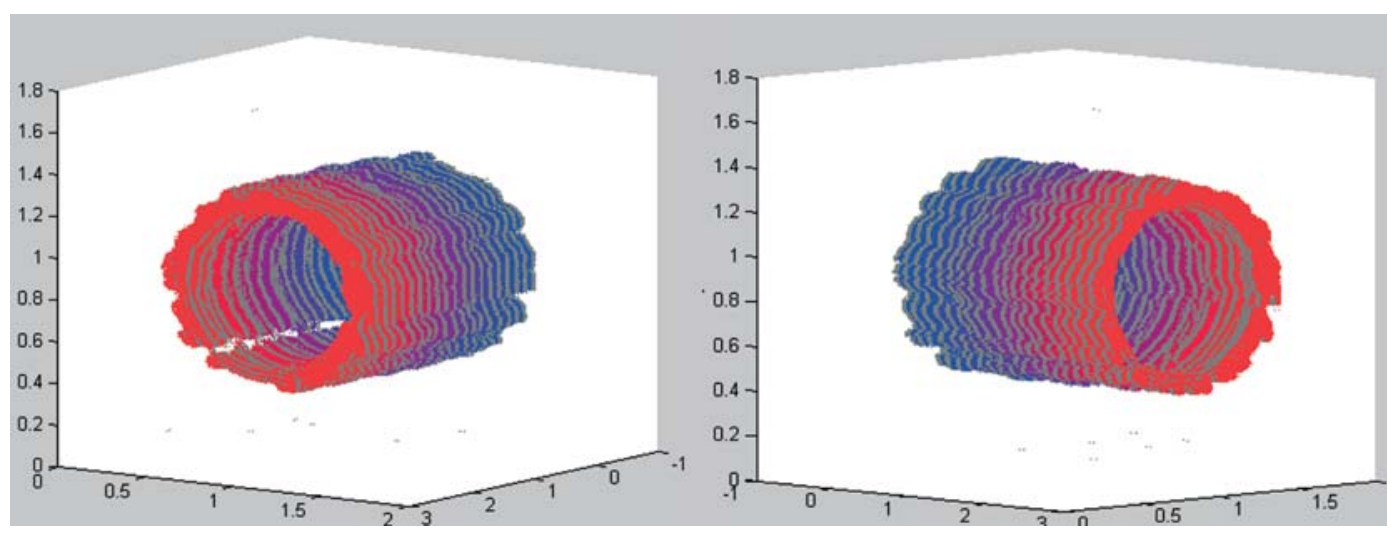

Figure 10: The sequence of images is combined into a montage, revealing how the shear layers at the periphery of the jet change with time. All of these images were obtained in a single plane, illuminated by the laser sheet.

Here, the two flow directions have been chosen to illustrate the principal features of the shear layer behaviour, both inside and outside the annular shear layer region.

Extensive studies over a long period have confirmed that the excitation generally increases the mixing of the turbulent free jet, so that it spreads more rapidly, and that there are preferred frequencies for the pulsations. Further information may be found in $[14,15,16]$. 


\section{EXPERIMENTAL STUDY OF THE FLOW OVER A HIGHLY SWEPT WING MODEL}

In some recent experiments to investigate the complex flow behaviour over a highly swept $40^{\circ}$ wing, the model was supported in the working section of a large wind tunnel. The basic arrangement is shown in Fig. 11. Observe, how colour has been employed, here, to emphasise key features of the test arrangements shown in the digital images, for example, the flow direction, the position and outline of the wing in the vertical plane, and the manner in which laser beams were directed into the flow through a window in the floor of the working section.

Visualisation of the boundary layer flow over the suction surface of the $40^{\circ}$ swept wing was also carried out, using a mixture of paraffin and green paint pigment - see also [17]. The wind tunnel was operated at a fixed speed until all the paint had been smeared across the surface by the airflow and all the paraffin had evaporated. The surface of the model was then photographed, using a combination of natural light and a camera flashlight, to produce images of the type shown in Fig. 12. These visualisation results were compared with measurements made very close to the surface (at a distance of only $0.2 \mathrm{~mm}$ ) using the three-component LDA system shown in Fig. 11. The calculated directions, derived from the three-component LDA measurements and denoted by the red arrows, are in excellent agreement with the flow visualisation images.

In Fig. 13a, the digital photograph of the aerofoil surface has been presented as a 'grey scale' with 256 grey levels (from white to black). In this form, there is plenty of detail showing the direction of the boundary layer flow (very close to the wing surface). In contrast, Figs 12 and 13b show the same experimental results, presented in the original colour of the green dye pigment that was exposed to the
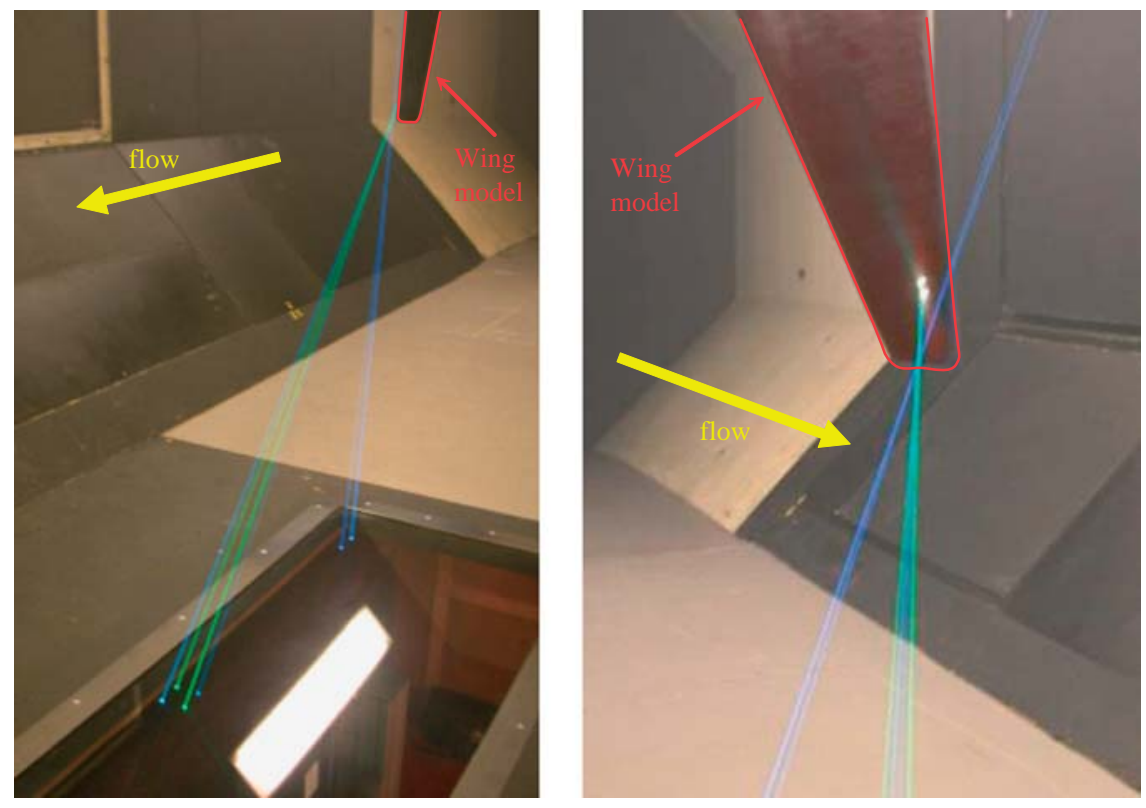

Figure 11: Arrangement of the $40^{\circ}$ wing in the vertical plane. The laser beams entered the working section from below, and the scattered light was collected downwards. The view on the left shows the intersection of the three pairs of (green, blue and violet) laser beams, with flow from right to left. The view on the right shows the location of the beam intersection (i.e. measurement volume) close to the wing surface, with flow from left to right. 


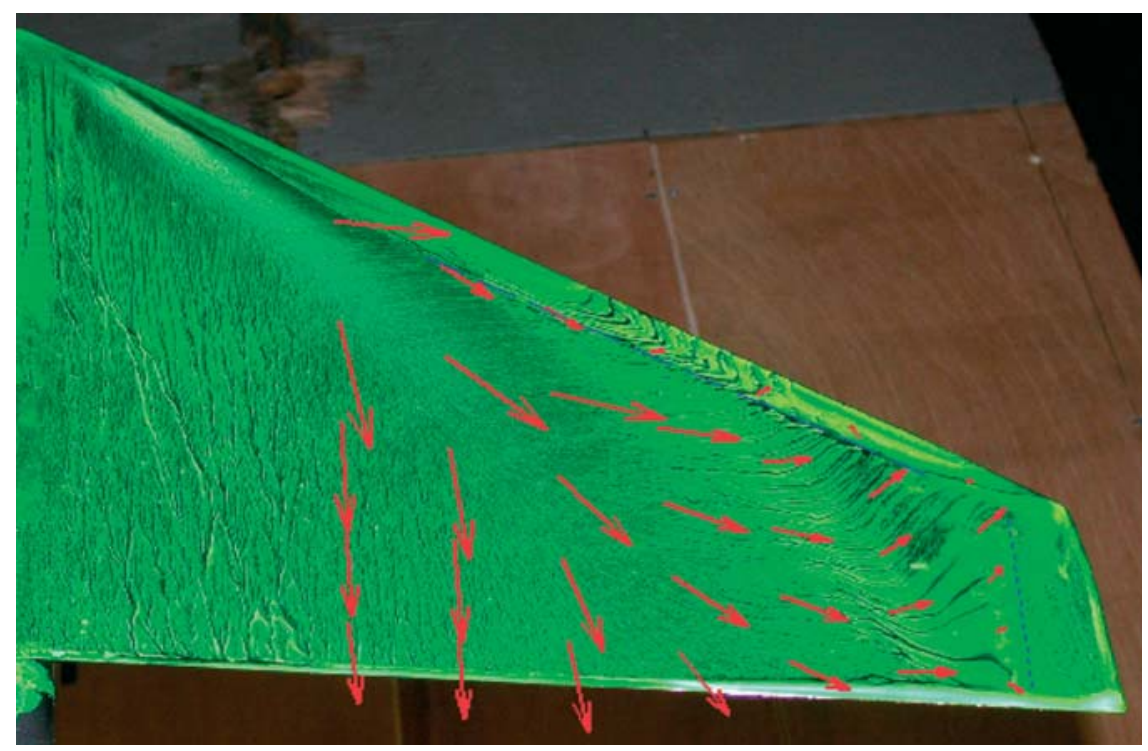

Figure 12: Typical flow visualisation results obtained for the $40^{\circ}$ swept wing, compared with velocity vectors derived from LDA measurements at $0.2 \mathrm{~mm}$ above the surface.

(a)

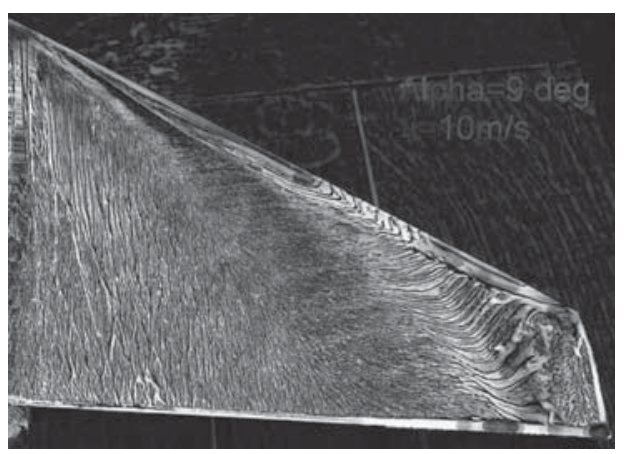

(b)

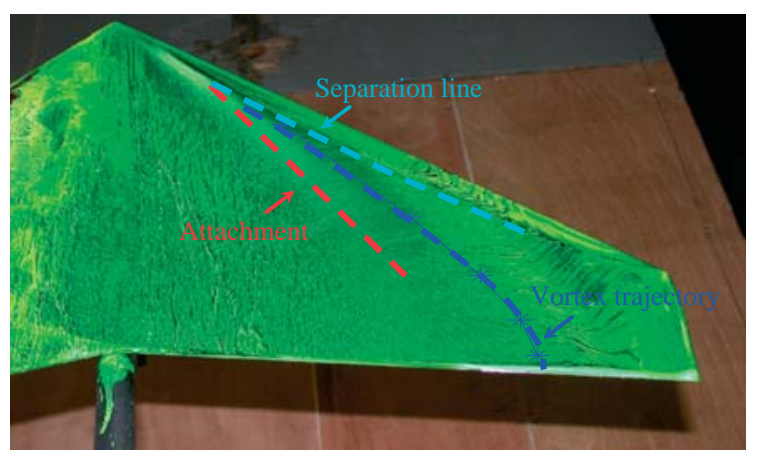

Figure 13: Comparison between grey scale and colour representations of the same digital photograph.

wind tunnel flow. These comparisons reveal the greater visual impact and, certainly, the more attractive appearance of the colour versions, although both types of image contain the same basic information.

An important feature of the flow over a wing and, particularly, a swept wing of the type considered here, is that some of the boundary layer flow rolls up to produce a vortex, which then trails away behind the wing. The low pressure region associated with the core of this vortex is responsible for the vapour trail often seen when an aeroplane passes overhead, or can be observed above a wing as the aeroplane approaches the runway. While this information could be gained from a close examination of the image shown in Figs. 12, 13, there are better ways of showing its existence. 


\section{THREE-DIMENSIONAL VIEWS OF EXPERIMENTAL DATA, COMBINING SEVERAL TYPES OF REPRESENTATION IN ONE SCHEMATIC}

Computer software now allows the production of complex shapes, images and graphical representations. These can be combined, to considerable advantage, as will be shown by the following example, which is based on the study of the swept wing.

The different areas of the surface flow shown in Fig. 12 were replaced by coloured contours, representing each velocity range by a different colour. These contours have then been combined with velocity measurements obtained using the LDA technique. One-thousand velocity measurements were made automatically under computer control at each traverse position and the average velocity values were the calculated so as to remove unsteadiness. Next, this velocity information was reduced to a set of contours, each range of velocities being represented by a particular colour. Combining these contours with the surface flow visualisation results discussed earlier enabled the schematic representation shown in Fig. 14 to be created.

Presenting the results in the form shown in Fig. 14 helps to clarify the complex flow behaviour over the wing surface, since the isometric view now combines the quantitative velocity information with the surface flow visualisation and a view of the swept wing. The direction of the flow is shown by the arrow in each view while the dashed line indicates the trajectory of the trailing vortex generated in the boundary layer flow. Predicting this locus, and the strength of the vortex, provides critical conditions against which numerical predictions can be evaluated. Another tremendously powerful feature of digital graphics is the ability to change the direction of viewing, on simple command from the mouse. This enables data to be viewed from several directions in succession so as to reveal any significant

(a)

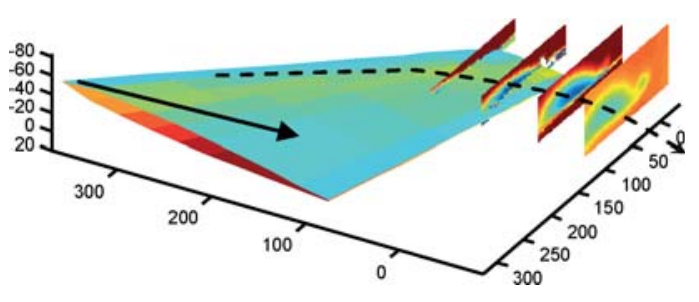

(c)

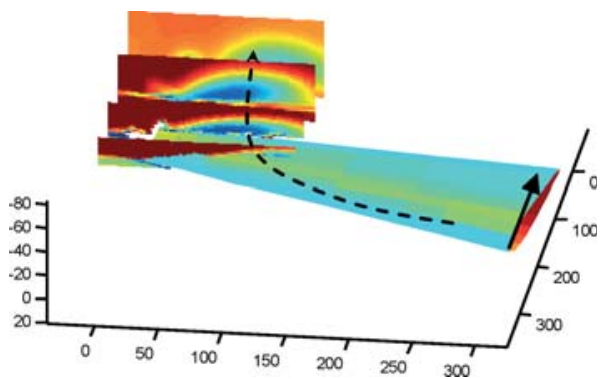

(b)

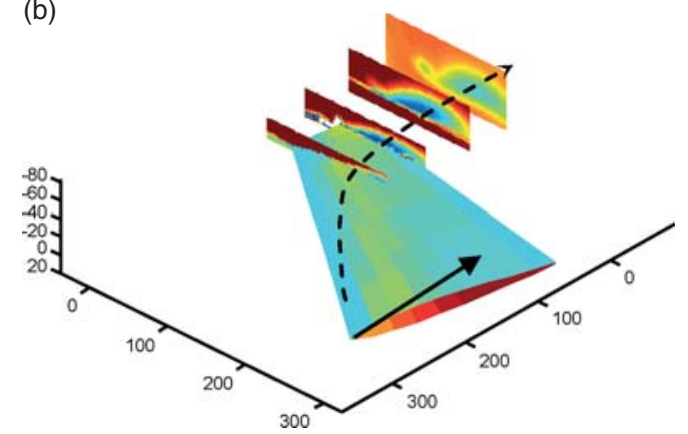

(d)

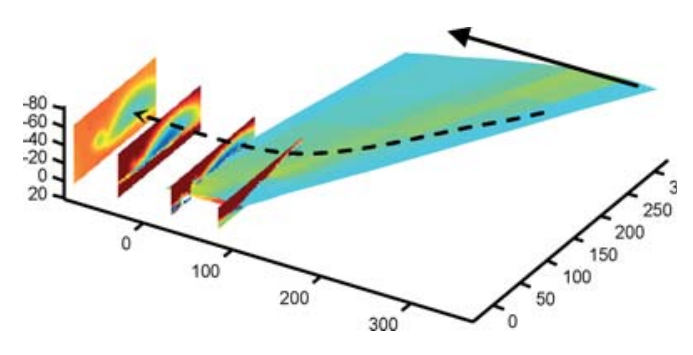

Figure 14: These isometric views combine a schematic of the $40^{\circ}$ swept wing with the velocity contours obtained by LDA measurement and the results of the surface flow visualisation. In this figure, the same data is viewed from four arbitrary directions. 
behaviour of the flow - although massive amounts of calculation are needed to achieve this. By way of example, the four views in Fig. 14 show the same graphical information at different orientations, thereby revealing how the vortex moves relative to the upper surface of the swept wing.

It is estimated that a minimum of $500 \mathrm{MB}$ of numerical data was needed to process the LDA and image data, before constructing just one of these views. Further vectorised information was then necessary to create the other different views as the images were rotated. Clearly, this computationally intensive process would never have been possible in the days before digital technology was available. It should also be noted, for the record, that the experimental results embodied in Fig. 14 correspond to tests performed in the wind tunnel at just one free-stream velocity level and one angle of incidence.

\section{CONCLUSIONS}

Colour discrimination is at the heart of many tools used by the experimentalist, providing a basis for the optical techniques that make use of some property of laser light to determine a flow characteristic and because of the advantages that colour offers for graphical representations and images. A number of these topics have been discussed and some examples of the possibilities have been given. It is the view of the authors that digital technology, and particularly non-intrusive optical methods of measurement, have revolutionised experimental fluid mechanics. It is surprising to reflect that these changes have taken place over a period of no more than thirty years.

\section{REFERENCES}

[1] Turner, J.T. \& Zhang, S., Analysis, presentation, and understanding in experimental fluid flow studies: an evolutionary story. J. Optics and Laser Technology. (doi: 10.1016/ j.optiastec.2009.12.002).

[2] For information on the work of Leonardo da Vinci. Available from: (a) www.efluids.com/ efluids/gallery/gallery_pages/da_vinci_page.htm; (b) http://en.wikipedia.org/wiki/Leonardo_ da_Vinci\#_Scientific_studies.

[3] Bertolotti, M., The History of the Laser, CRC Press, 2005.

[4] For the invention of the laser. Available from: www.bell-labs.com/history/laser/. Additionally, regarding the disputed ownership of this invention. Available from: http://www.essortment. com/all/historylaserin_rnxv.htm.

[5] Yeh, Y. \& Cummins, H.Z., Localized fluid flow measurements with a He-Ne laser spectrometer. Applied Physics Letters, 4, p. 176, 1964.

[6] Durst, F., Melling, A. \& Whitelaw, J.H., Principles and Practice of Laser-Doppler Anemometry, Academic Press, 1976.

[7] Albrecht, H.E., Borys, M., Damaschke, N. \& Tropea, C., Laser Doppler and Phase Doppler Measurement Techniques, Springer-Verlag, 2003.

[8] Adrian, R.J., Twenty years of particle image velocimetry. Experiments in Fluids, 39(2), pp. 159-169, 2005. doi:10.1007/s00348-005-0991-7

[9] For alternative methods of colour representation, the definition of a pixel, and information on the colour and spatial responses of the human eye. Available from: (a) http://www.klammeraffe. org/ fritsch/uni-sb/fsinfo/Papers/webdesign/webdesign.html; (b) http://en.wikipedia.org/wiki/ Color_vision; (c) http://www.stanford.edu/class/ee368b/Handouts/09-HumanPerception.pdf.

[10] Hiller, W. \& Kowalewski, T.A., Simultaneous measurement of the temperature and velocity fields in thermal convective flows, in Flow Visualization IV, ed C. Veret, Hemisphere: Paris, pp. 617-622, 1987.

[11] For an informative introduction to particle image velocimetry (PIV). Available from: www. holomap.com/dpiv.htm. 
[12] Papadopoulou, K.A., Shamout, M.N., Lennox, B., Mackay, D., Taylor, A.R., Turner, J.T. \& Wang, X., An evaluation of acoustic reflectometry for leakage and blockage detection. I.Mech.E. Proc., Series E, 222(6), pp. 959-966, 2008.

[13] Papadopoulou, K.A., Leakage and Blockage Detection in Pipelines Using an Acoustic Inspection Tool, PhD thesis, University of Manchester, 2008.

[14] Zhang, S., Digital imaging and optical flow diagnostics applied to turbulent jets with and without excitation, PhD thesis, University of Manchester, 2006.

[15] Szajner, A. \& Turner, J.T., Visualisation of an aerodynamically excited free jet, in Flow Visualization IV, ed C. Veret, Hemisphere: Paris, pp. 533-539, 1987.

[16] Zhang, S. \& Turner, J.T., Aerodynamic control of a free turbulent jet using helical excitation. Eighth International Symposium on Fluid Control, Measurement and Visualization (Flucome). Chengdu, China, 2005.

[17] Merzkirch, W., Flow Visualization, Academic Press: New York, 1987. 